\title{
Management of a Submacular Hemorrhage Secondary to Age-Related Macular Degeneration: A Comparison of Three Treatment Modalities
}

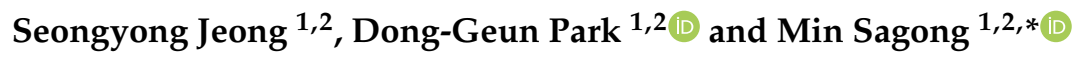 \\ 1 Department of Ophthalmology, Yeungnam University College of Medicine, Daegu 42415, Korea; \\ jjsssyyyy@naver.com (S.J.); bluepdg@naver.com (D.-G.P.) \\ 2 Yeungnam Eye Center, Yeungnam University Hospital, Daegu 42415, Korea \\ * Correspondence: msagong@ynu.ac.kr; Tel.: +82-53-620-3443
}

Received: 24 August 2020; Accepted: 23 September 2020; Published: 24 September 2020

\begin{abstract}
This paper aims to compare the effects of three treatment modalities for a submacular hemorrhage (SMH) secondary to exudative age-related macular degeneration (AMD). Seventy-seven patients with an SMH were divided into three groups: small-sized (optic disc diameter (ODD) $\geq 1$ to $<4$ ), medium-sized (ODD $\geq 4$ within the temporal arcade) and large-sized (ODD $\geq 4$, exceeding the temporal arcade). Patients received anti-vascular endothelial growth factor (anti-VEGF) monotherapy, pneumatic displacement (PD) with anti-VEGF or a vitrectomy with a subretinal tissue plasminogen activator (tPA) and gas tamponade based on the surgeon's discretion. The functional and anatomical outcomes were evaluated. Among the 77 eyes, 45 eyes had a small-sized, 21 eyes had a medium-sized and 11 eyes had a large-sized SMH. In the small-sized group, all treatment modalities showed a gradual best-corrected visual acuity (BCVA) improvement with high hemorrhagic regression or displacement rates (over $75 \%$ ). In the medium-sized group, PD and surgery were associated with better BCVA with more displacement than anti-VEGF monotherapy (67\% and $83 \%$, respectively, vs. $33 \%)$. In the large-sized group, surgery showed a better visual improvement with a higher displacement rate than PD $(86 \%$ vs. $25 \%)$. Our findings demonstrated that visual improvement can be expected through appropriate treatment strategy regardless of the SMH size. In cases with a larger SMH, invasive techniques including PD or surgery were more advantageous than anti-VEGF monotherapy.
\end{abstract}

Keywords: age-related macular degeneration; anti-VEGF; pneumatic displacement; submacular hemorrhage; tissue plasminogen activator

\section{Introduction}

Submacular hemorrhage (SMH) is one of the severe complications in patients with neovascular age-related macular degeneration (AMD). As it physically separates the neurosensory retina from the retinal pigment epithelium (RPE) layer, the resulting barrier effect interferes with the diffusion of nutrients and metabolites [1]. Additionally, the shearing of the photoreceptor from enmeshed fibrin and iron toxicity in the retina due to the blood cause photoreceptor death leading to a poor visual prognosis $[1,2]$.

Various treatment modalities for SMH have been developed. Expansile gas injection including perfluoropropane $\left(\mathrm{C}_{3} \mathrm{~F}_{8}\right)$ or sulfahexafluoride $\left(\mathrm{SF}_{6}\right)$ can be used to push away the $\mathrm{SMH}$ from the fovea. A tissue plasminogen activator (tPA), a serine protease, dissolves the blood clot by cleaving the fibrin [3]. Anti-vascular endothelial growth factor (anti-VEGF) drugs affect the underlying choroidal neovascularization (CNV) and treat the SMH [4]. A surgical intervention, pars plana vitrectomy (PPV), facilitates vitreous oxygenation and reduces the VEGF level [5]. Single or combined modalities have been used to treat the SMH. 
A widely used treatment option for the SMH is anti-VEGF monotherapy. It has shown favorable results but severe cases were often excluded from previous studies [6-9]. Thick blood under the fovea can be displaced by the tPA and subsequent expansile gas. However, when patients received high concentrations of the tPA, i.e., over $100 \mu \mathrm{g}$, retinal toxicity could occur [10]; its limited efficacy has been reported in previous studies [11,12]. A retinal tear or detachment could be observed after intravitreal gas injection [13]. Moreover, there are cases in which a tPA with gas injection should be avoided such as in patients with superior-juxtafoveal SMH and those who cannot maintain a prone position [14]. Although surgical treatment with PPV, a subretinal tPA and expansile gas have been reported to provide better visual acuity, the procedure is more complex and needs additional special instruments such as a 41-gauge cannula. Therefore, an appropriate treatment selection per case may be essential for patients with an SMH. To date, the rarity of the disease [15] and devasting prognosis [16] have limited randomized clinical trials and there is a lack of consensus on the standard treatment of patients with AMD complicated by an SMH.

Hence, we sought to compare the anatomical and functional outcomes of practically selected treatment modalities depending on the hemorrhage size in patients with AMD complicated by an $\mathrm{SMH}$. We also evaluated the possible prognostic factors for visual outcomes.

\section{Materials and Methods}

A retrospective chart review of patients with an SMH from March 2015 to November 2018 at Yeungnam University Hospital was performed. The Institutional Review Board of the Yeungnam University Hospital approved the protocol. Data were collected from medical charts of patients who had an SMH involving the fovea secondary to exudative AMD. Additional inclusion criteria were age $>50$ years and SMH thickness $>250 \mu \mathrm{m}$.

The baseline evaluation included best-corrected visual acuity (BCVA) with a Snellen chart, dilated fundus examination, fundus photography and spectral domain optical coherence tomography (SD-OCT). An angiography with fluorescein or indocyanine green was performed to identify the underlying lesion.

The fundus images were evaluated with ImageJ software (National Institutes of Health, Bethesda, MD) for Windows. The longest diameter of the SMH was measured and recorded as multiple values of the optic disc diameter (ODD). Patients were divided into three groups based on the size of the SMH: small-sized ( $\geq 1$ to $<4$-disc ODD), medium-sized ( $\geq 4$ ODD and not extending beyond the temporal arcade) and large-sized ( $\geq 4$ ODD and extending beyond the temporal arcade) [5]. The different treatment outcomes among the subgroups were compared.

Using SD-OCT (Spectralis; Heidelberg Engineering, Heidelberg, Germany), a standard volume scan consisting of a $6 \times 6 \mathrm{~mm}$ area was performed at the macular area and at points considered to have the largest SMH. A retinal specialist (Dong-Geun Park) reviewed all the images and measured the maximum height of the SMH using a manual ruler in the integrated software.

Patients were prescribed a treatment modality based on the surgeon's judgement of optimal treatment. Twenty-nine patients were treated with an intravitreal anti-VEGF monotherapy (bevacizumab, ranibizumab or aflibercept). Twenty-five patients received pneumatic displacement (PD) using $0.3 \mathrm{cc}$ of $100 \% \mathrm{C}_{3} \mathrm{~F}_{8}$ gas with an intravitreal anti-VEGF injection simultaneously with or without an intravitreal tPA. When anti-VEGF therapy was combined with the tPA, aflibercept, which is known to be cleaved by the tPA [17], was avoided. Twenty-three patients underwent a 25-gauge PPV followed by a subretinal tPA (Actilyse, Boehringer Ingelheim, Germany) injection with a 41-gauge flexible needle (De Juan/Awh Subretinal Injection Cannula, Synergetics Inc, USA) and a $20 \% \mathrm{SF}_{6}$ gas filling through a fluid-gas exchange.

The surgeon applied these three strategies according to the SMH size. Anti-VEGF monotherapy was considered for the small-sized SMH preferentially. $\mathrm{C}_{3} \mathrm{~F}_{8}$ gas with an anti-VEGF injection $\left(\mathrm{C}_{3} \mathrm{~F}_{8} /\right.$ anti-VEGF $)$ was selected mainly for the medium-sized $\mathrm{SMH}$ and a PPV with a subretinal $\mathrm{tPA}$ and $\mathrm{SF}_{6}$ gas (PPV/tPA/ $\mathrm{SF}_{6}$ ) was preferred for the large-sized $\mathrm{SMH}$, respectively. Additionally, parameters including location, 
thickness and duration of the hemorrhage were considered to achieve the best outcomes for each patient when choosing the treatment option. Every procedure was performed by a retinal surgeon (Min Sagong). Patients who received any gas injections were instructed to maintain a face-down position for 2-3 days.

If a patient complained of decreased vision during the follow-up period, we performed thorough examinations to detect any sign of recurrence including a newly developed $\mathrm{SMH}$, a small retinal hemorrhage, increased retinal thickness on an OCT, leakage on an angiography or decreased vision alone. Based on the surgeon's decision, additional anti-VEGF injections were administered.

The main outcomes were the degree of displacement or regression of the SMH from the fovea at 1 month after treatment; BCVA at 1, 3, 6 and 12 months after the initial treatment; prognostic factors for visual acuity; frequency of additional anti-VEGF injections; incidence of a recurrent SMH or breakthrough vitreous hemorrhage and other complications.

Statistical analyses were performed using IBM SPSS ver. 20.0 for Windows (IBM Co., Armonk, New York, USA). BCVA was measured using a Snellen chart and converted to the logarithm of the minimum angle of resolution (logMAR) units for statistical analyses. An analysis of variance or a Kruskal-Wallis test was performed to determine the differences in the baseline parameters among the subgroups. If there was a difference, a post-hoc analysis with a Bonferroni correction was performed to identify the groups that caused that significance. Categorical variables among the subgroups or among the treatment modalities were analyzed by $\chi 2$ test or Fisher's exact test. To identify factors that correlated with BCVA at 3 months and 12 months, a Pearson correlation test and analysis of covariance (ANCOVA) were performed. Variables with a $p$-value $<0.05$ were considered significant.

\section{Results}

\subsection{Baseline Characteristics}

Among the 77 eyes of 77 patients with hemorrhagic AMD, 45 eyes had a small-sized, 21 eyes had a medium-sized and 11 eyes had a large-sized SMH. The baseline characteristics of the affected eyes are provided in Table 1. Eyes in three groups did not show a difference in terms of age $(p=0.164)$, $\operatorname{sex}(p=0.755)$, duration of symptoms $(p=0.653)$, lens status $(p=0.119)$ and proportion of anticoagulant usage $(p=0.068)$. The mean size of the SMH as an ODD was $2.45 \pm 0.90,5.09 \pm 1.27$ and $7.55 \pm 2.27$ in the small-, medium- and large-sized subgroups $(p<0.001)$, respectively. The mean SMH thickness in each group increased according to the SMH size $(474.6 \pm 149.6,791.9 \pm 482.0$ and $955.4 \pm 375.9 \mu \mathrm{m}$, respectively; $p<0.001)$. Regarding the AMD lesions, the small-sized group showed a higher percentage of polypoidal choroidal vasculopathy (PCV) (66.7\%) than CNV (33.3\%). In contrast, the large-sized group consisted of more CNV (72.7\%) than PCV (27.3\%). There was a statistical difference in the distribution of classifications among the three groups $(p=0.028)$. The treatment modalities were differently distributed in each subgroup $(p=0.011)$. Anti-VEGF monotherapy was applied mainly in the small-sized group $(51.1 \%)$ and also applied in the medium-sized (28.6\%) groups. A combination of PD with anti-VEGF and/or an intravitreal tPA injection was applied in all subgroups (26.7\% in the small-, $42.8 \%$ in the medium- and $36.4 \%$ in the large-sized groups). A PPV with a subretinal $\mathrm{tPA}$ and $\mathrm{SF}_{6}$ gas, although also applied in the small- $(22.2 \%)$ and medium-sized (28.6\%) groups, was mainly applied in the large-sized group (63.6\%).

\subsection{SMH Displacement or Regression Rate}

The distribution of the SMH displacement did not significantly differ among the subgroups $(p=0.200$; Table 2). All three groups achieved a relatively high percentage of complete SMH displacement or regression $(82 \%, 62 \%$ and $73 \%$, respectively); however, it was achieved by different modalities in each group. In the small-sized group, anti-VEGF monotherapy achieved $78 \%$ of the complete $\mathrm{SMH}$ regression and $\mathrm{C}_{3} \mathrm{~F}_{8} /$ anti-VEGF and a PPV/tPA/SF $\mathrm{SF}_{6}$ showed $75 \%$ and $100 \%$ of complete displacement, respectively. In the medium-sized group, anti-VEGF monotherapy showed poor anatomical outcomes ( $33 \%$ of complete regression) while the other two treatment methods showed acceptable results (67\% and $83 \%$ of complete displacement, respectively). In the large-sized group, 
the PPV/tPA/SF 6 contributed to the high percentage of complete displacement $(86 \%)$ but $\mathrm{C}_{3} \mathrm{~F}_{8} /$ anti-VEGF $\operatorname{did} \operatorname{not}(25 \%)$ (Table 2).

Table 1. Baseline Characteristics.

\begin{tabular}{|c|c|c|c|c|c|}
\hline & Small-Sized SMH & Medium-Sized SMH & Large-Size dSMH & $p$-Value & Total \\
\hline No. of patients & 45 & 21 & 11 & & 77 \\
\hline Mean age, years & $71.5 \pm 10.9$ & $74.8 \pm 11.1$ & $72.3 \pm 9.0$ & $0.164^{\mathrm{a}}$ & $73.2 \pm 10.8$ \\
\hline $\operatorname{Sex}(\%)$ & & & & $0.755^{b}$ & \\
\hline Female & $12(26.7)$ & $7(33.3)$ & $4(36.4)$ & & $23(29.9)$ \\
\hline Systemic anticoagulants (\%) & $9(20.0)$ & $10(47.6)$ & $3(27.4)$ & $0.068^{b}$ & $22(100.0)$ \\
\hline Duration of SMH, days & $16.6 \pm 30.6$ & $10.6 \pm 19.7$ & $12.4 \pm 11.9$ & $0.653^{\mathrm{a}}$ & $14.3 \pm 25.8$ \\
\hline Classification of lesions (\%) & & & & $0.028^{b}$ & \\
\hline $\mathrm{CNV}$ & $15(33.3)$ & $12(57.1)$ & $8(72.7)$ & & $35(45.5)$ \\
\hline PCV & $30(66.7)$ & $9(42.9)$ & $3(27.3)$ & & $42(54.5)$ \\
\hline Preoperative anti-VEGF treatment & $2(4.4)$ & $3(14.3)$ & $5(45.5)$ & $0.005^{b}$ & $10(13.0)$ \\
\hline Lens status $(\%)$ & & & & $0.119^{b}$ & \\
\hline Phakic & $40(88.9)$ & $14(66.7)$ & $8(72.7)$ & & $62(80.5)$ \\
\hline $\begin{array}{c}\text { Intravitreal anti-VEGF with } \mathrm{C}_{3} \mathrm{~F}_{8} \\
\text { gas }\end{array}$ & $12(26.7)$ & $9(42.8)$ & $4(36.4)$ & & 25 (32.5) \\
\hline $\begin{array}{c}\text { PPV with subretinal tPA and } \mathrm{SF}_{6} \\
\text { gas }\end{array}$ & $10(22.2)$ & $6(28.6)$ & 7 (63.6) & & $23(29.8)$ \\
\hline
\end{tabular}

Values are presented as mean \pm SD unless indicated otherwise; ${ }^{a} p$-value using analysis of variance or a Kruskal-Wallis test; ${ }^{\mathrm{b}} p$-value using $\chi^{2}$ test or Fisher's exact test; $\mathrm{C}_{3} \mathrm{~F}_{8}$, perfluoropropane; $\mathrm{CNV}$, choroidal neovascularization; ODD, optic disc diameter; PCV, polypoidal choroidal vasculopathy; PPV, pars plana vitrectomy; $\mathrm{SF}_{6}$, sulfahexafluoride; $\mathrm{SMH}$, submacular hemorrhage; tPA, tissue plasminogen activator; VEGF, vascular endothelial growth factor.

Table 2. Functional and anatomical outcomes.

\begin{tabular}{|c|c|c|c|c|c|}
\hline & Small-Sized SMH & Medium-Sized SMH & Large-Sized SMH & $p$-Value & Total \\
\hline \multicolumn{6}{|l|}{ LogMAR BCVA } \\
\hline Baseline $(n=77)$ & $1.06 \pm 0.70$ & $1.5 \pm 0.89$ & $1.93 \pm 0.84$ & $0.015^{\mathrm{a}}$ & $1.30 \pm 0.83$ \\
\hline 1 month $(n=77)$ & $0.83 \pm 0.55$ & $1.33 \pm 0.83$ & $2.45 \pm 0.94$ & $0.002^{\mathrm{a}}$ & $1.19 \pm 0.87$ \\
\hline 6 months $(n=51)$ & $0.59 \pm 0.56$ & $1.24 \pm 0.90$ & $1.84 \pm 1.11$ & $0.145^{\mathrm{a}}$ & $1.00 \pm 0.93$ \\
\hline 12 months $(n=41)$ & $0.41 \pm 0.45$ & $1.14 \pm 0.85$ & $1.52 \pm 0.89$ & $0.139^{\mathrm{a}}$ & $0.76 \pm 0.83$ \\
\hline Mean follow-up period, months & $17.8 \pm 19.4$ & $17.7 \pm 15.1$ & $16.2 \pm 12.9$ & $0.963^{\mathrm{a}}$ & $16.2 \pm 17.4$ \\
\hline Anti-VEGF monotherapy & $18 / 23(78.3)$ & $2 / 6(33.3)$ & - & & $20 / 29(69.0)$ \\
\hline Intravitreal anti-VEGF with $\mathrm{C}_{3} \mathrm{~F}_{8}$ gas & $9 / 12(75.0)$ & $6 / 9(66.7)$ & $1 / 4(25.0)$ & & $16 / 25(64.0)$ \\
\hline $\begin{array}{c}\text { PPV with a subretinal tPA and } \mathrm{SF}_{6} \text { gas } \\
\text { Additional intravitreal anti-VEGF } \\
\text { injections }\end{array}$ & $10 / 10(100.0)$ & $5 / 6(83.3)$ & $6 / 7(85.7)$ & & $21 / 23(91.3)$ \\
\hline No. of eyes treated with anti-VEGF (\%) & $30(66.7)$ & $15(71.4)$ & $8(72.7)$ & $0.886^{\mathrm{b}}$ & $53(68.8)$ \\
\hline RPE rip & 0 & 1 & 0 & & 1 \\
\hline Macular hole & 0 & 1 & 0 & & 1 \\
\hline Retinal detachment & 0 & 0 & 0 & & 0 \\
\hline
\end{tabular}

Values are presented as mean $\pm \mathrm{SD}$ unless indicated otherwise; ${ }^{\mathrm{a}} p$-values indicate the significance of difference among the subgroups using analysis of variance or a Kruskal-Wallis test; ${ }^{b} p$-values using $\chi^{2}$ test or Fisher's exact test; $\mathrm{BCVA}$, best-corrected visual acuity; $\mathrm{C}_{3} \mathrm{~F}_{8}$, perfluoropropane; LogMAR, logarithm of the minimum angle of resolution; $\mathrm{PPV}$, pars plana vitrectomy; $\mathrm{RPE}$, retinal pigment epithelium; $\mathrm{SF}_{6}$, sulfahexafluoride; $\mathrm{SMH}$, submacular hemorrhage; tPA, tissue plasminogen activator; VEGF, vascular endothelial growth factor.

\subsection{Visual Outcomes}

The baseline mean BCVA differed among the groups $(p=0.015)$. The post-hoc analysis revealed a significant effect of the large-sized group when compared with that of the small-sized group $(p=0.014)$. The overall mean $\log$ MAR BCVA improved from $1.30 \pm 0.83$ at baseline to $1.03 \pm 0.95$ at 3 months 
$(p=0.040), 1.00 \pm 0.93$ at 6 months $(p=0.044)$ and $0.76 \pm 0.83$ at 12 months $(p=0.008)$. Each subgroup showed an improvement of mean BCVA at each follow-up; the difference in the baseline mean BCVA among the subgroups disappeared at 6 months $(p=0.145)$ and 12 months $(p=0.139)$ (Table 2).

In the subgroup analyses, the small-sized group showed an improved mean BCVA regardless of the treatment modality. In the medium-sized group, anti-VEGF monotherapy had little effect on the visual improvement while the other modalities improved the mean BCVA for 12 months. In the large-sized group, the surgeon excluded anti-VEGF monotherapy. $\mathrm{C}_{3} \mathrm{~F}_{8} /$ anti-VEGF was used in $36.4 \%$ and a PPV/tPA/SF $/ \mathrm{SF}_{6}$ was employed in $63.6 \%$ of patients. The mean BCVA improved only in the $\mathrm{PPV} / \mathrm{tPA} / \mathrm{SF}_{6}$ group and decreased in the $\mathrm{C}_{3} \mathrm{~F}_{8}$ /anti-VEGF group (Table 3; Figure 1).

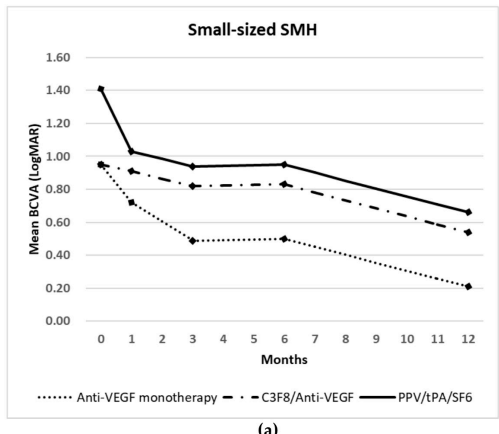

(a)

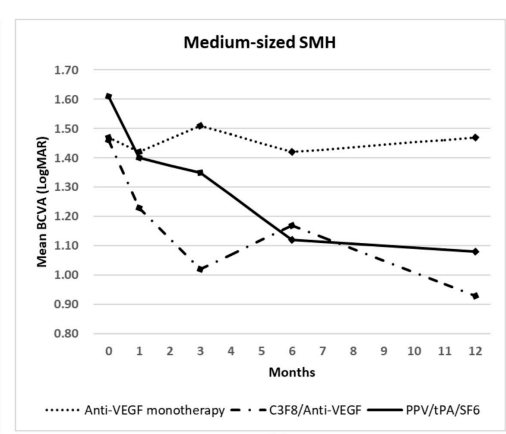

(b)

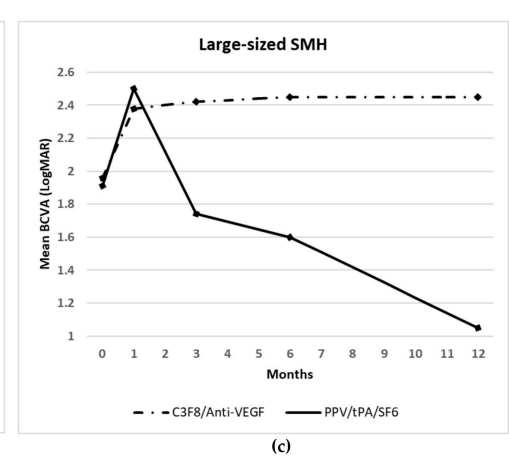

Figure 1. Changes in the mean best-corrected visual acuity (BCVA) in the (a) small-, (b) medium- and (c) large-sized submacular hemorrhage (SMH) groups. The eyes were treated with anti-vascular endothelial growth factor (anti-VEGF) monotherapy, pneumatic displacement (PD) using perfluoropropane $\left(\mathrm{C}_{3} \mathrm{~F}_{8}\right)$ with an anti-VEGF injection or a pars plana vitrectomy (PPV) with a subretinal tissue plasminogen activator (tPA) and gas tamponade using sulfahexafluoride $\left(\mathrm{SF}_{6}\right)$. $\mathrm{C}_{3} \mathrm{~F}_{8} / \mathrm{Anti-VEGF}$, PD with anti-VEGF; LogMAR, logarithm of the minimal angle of resolution; $\mathrm{PPV} / \mathrm{tPA} / \mathrm{SF}_{6}, \mathrm{PPV}$ with subretinal tPA and gas tamponade.

\subsection{Factors Associated with Visual Outcomes}

A significant positive correlation was identified between the BCVA at baseline and 3 months $(r=0.543, p<0.001)$ and between the BCVA at 3 months and 12 months $(r=0.875, p<0.001)$. The SMH thickness also showed a positive correlation with BCVA at 3 months $(r=0.592, p<0.001)$ and 12 months $(r=0.497, p=0.001 ;$ Figure 2).

To identify the effect of treatment modality on visual acuity during the follow-up, ANCOVA with baseline visual acuity as a covariance was performed, which is a known prognostic factor. However, different treatment modalities did not influence the visual outcomes. We divided patients into two groups based on the SMH duration (duration $\leq 14$ days or $>14$ days) and performed ANCOVA with baseline visual acuity as a covariant. There was a significant difference in visual outcomes $(p=0.022$ at 3 months and $p=0.022$ at 12 months) between the two groups.

\subsection{Frequency of Additional Anti-VEGF Injections}

For 12 months, additional anti-VEGF injections were administered to $66.7 \%, 71.4 \%$ and $72.7 \%$ of eyes in each group (Table 2$)$. There was no statistical significance $(p=0.886)$. The mean number of injections were $3.27 \pm 2.06,2.60 \pm 1.45$ and $3.00 \pm 1.85$ during 12 months in each group $(p=0.526)$.

\subsection{Complications}

Breakthrough vitreous hemorrhage occurred in three $(6.7 \%)$, one $(4.8 \%)$ and four $(36.4 \%)$ eyes of each group. One case of a recurrent $\mathrm{SMH}$, one case of an RPE rip and one case of a macular hole were observed in the medium-sized group. (Table 2). The RPE rip and macular hole were associated with the PPV/tPA/SF 6 . 
Table 3. Subgroup analysis of treatment outcomes based on SMH size.

\begin{tabular}{|c|c|c|c|c|c|c|c|c|c|c|c|}
\hline & \multicolumn{4}{|c|}{ Small-Sized SMH Group } & \multicolumn{4}{|c|}{ Medium-Sized SMH Group } & \multicolumn{3}{|c|}{ Large-Sized SMH Group } \\
\hline & $\begin{array}{c}\text { Anti-VEGF } \\
\text { Monotherapy } \\
(n=23)\end{array}$ & $\begin{array}{c}\mathrm{C}_{3} \mathrm{~F}_{8} / \text { Anti-VEGF } \\
(n=12)\end{array}$ & $\begin{array}{c}\mathrm{PPV} / \mathrm{tPA} / \mathrm{SF}_{6} \\
(n=10)\end{array}$ & $p$-Value & $\begin{array}{c}\text { Anti-VEGF } \\
\text { Monotherapy } \\
(n=6)\end{array}$ & $\begin{array}{c}\mathrm{C}_{3} \mathrm{~F}_{8} / \text { Anti-VEGF } \\
(n=9)\end{array}$ & $\begin{array}{c}\mathrm{PPV} / \mathrm{tPA} / \mathrm{SF}_{6} \\
(n=6)\end{array}$ & $p$-Value & $\begin{array}{c}\mathrm{C}_{3} \mathrm{~F}_{8} / \text { Anti-VEGF } \\
(n=4)\end{array}$ & $\begin{array}{c}\mathrm{PPV} / \mathrm{tPA} / \mathrm{SF}_{6} \\
(n=7)\end{array}$ & $p$-Value \\
\hline Mean size of SMH, ODD & $2.00 \pm 0.80$ & $2.56 \pm 0.70$ & $3.34 \pm 0.59$ & $0.001^{\mathrm{a}}$ & $5.93 \pm 2.00$ & $4.73 \pm 0.84$ & $4.77 \pm 0.39$ & $0.148^{\mathrm{a}}$ & $8.46 \pm 2.23$ & $7.03 \pm 2.30$ & $0.345^{\mathrm{b}}$ \\
\hline $\begin{array}{c}\text { Mean thickness of SMH, } \\
\mu \mathrm{m}\end{array}$ & $431.6 \pm 131.0$ & $541.4 \pm 194.7$ & $493.3 \pm 100.7$ & $0.088^{a}$ & $669.6 \pm 470.0$ & $794.6 \pm 420.3$ & $910.0 \pm 625.2$ & $0.551^{\text {a }}$ & $1006.4 \pm 259.6$ & $921.4 \pm 458.7$ & $0.450^{\mathrm{b}}$ \\
\hline $\begin{array}{l}\text { Duration of SMH, days } \\
\text { LogMAR visual acuity } \\
\text { (No.) }\end{array}$ & $21.4 \pm 39.1$ & $8.3 \pm 9.2$ & $16.4 \pm 27.3$ & $0.370^{\mathrm{a}}$ & $28.6 \pm 35.8$ & $4.7 \pm 1.9$ & $4.5 \pm 3.4$ & 0.067 & $17.8 \pm 14.2$ & $9.3 \pm 10.2$ & $0.214^{\mathrm{b}}$ \\
\hline Baseline & $0.95 \pm 0.62(23)$ & $0.95 \pm 0.59(12)$ & $1.41 \pm 0.89(10)$ & $0.282^{\mathrm{a}}$ & $1.47 \pm 1.22(6)$ & $1.46 \pm 0.93(9)$ & $1.61 \pm 0.49(6)$ & $0.703^{\mathrm{a}}$ & $1.96 \pm 1.09(4)$ & $1.91 \pm 0.76(7)$ & $0.923^{b}$ \\
\hline 1 month & $0.72 \pm 0.57(23)$ & $0.91 \pm 0.62(12)$ & $1.03 \pm 0.31(10)$ & $0.036^{\mathrm{a}}$ & $1.42 \pm 1.18(6)$ & $1.23 \pm 0.85$ & $1.40 \pm 0.39(6)$ & $0.756^{\mathrm{a}}$ & $2.38 \pm 1.03(4)$ & $2.50 \pm 0.97(7)$ & $0.879^{b}$ \\
\hline 3 months & $0.49 \pm 0.41(20)$ & $0.82 \pm 0.77(9)$ & $0.99 \pm 0.85$ & $0.266^{\mathrm{a}}$ & $1.51 \pm 1.30$ & $1.02 \pm 0.66(7)$ & $1.35 \pm 0.47(5)$ & $0.679^{\mathrm{a}}$ & $2.42 \pm 0.95(4)$ & $1.74 \pm 1.13(5)$ & $0.421^{b}$ \\
\hline 6 months & $0.50 \pm 0.45(16)$ & $0.73 \pm 0.65(6)$ & $0.71 \pm 0.79(6)$ & $0.850^{\mathrm{a}}$ & $1.42 \pm 1.39$ & $1.17 \pm 0.70(6)$ & $1.12 \pm 0.54$ & $0.779^{\mathrm{a}}$ & $2.45 \pm 0.63(3)$ & $1.60 \pm 1.22(5)$ & $0.417^{b}$ \\
\hline 12 months & $0.21 \pm 0.22(10)$ & $0.39 \pm 0.41(6)$ & $0.83 \pm 0.62$ & $0.209^{\mathrm{a}}$ & $1.47 \pm 1.25$ & $0.93 \pm 0.75$ & $1.08 \pm 0.62(4)$ & $0.818^{\mathrm{a}}$ & $2.45 \pm 0.95(3)$ & $1.05 \pm 0.58$ & $0.064^{b}$ \\
\hline $\begin{array}{l}\text { Mean follow-up period, } \\
\text { months }\end{array}$ & $16.3 \pm 15.6$ & $15.3 \pm 19.9$ & $24.1 \pm 26.6$ & $0.512^{\mathrm{a}}$ & $20.3 \pm 20.9$ & $14.3 \pm 13.0$ & $20.2 \pm 12.8$ & $0.695^{\mathrm{a}}$ & $18.0 \pm 13.7$ & $15.1 \pm 13.4$ & $0.744^{\mathrm{b}}$ \\
\hline
\end{tabular}

months

${ }^{\mathrm{b}} p$-values using a Mann-Whitney test; $\mathrm{C}_{3} \mathrm{~F}_{8}$, perfluoropropane; LogMAR, logarithm of the minimum angle of resolution; ODD, optic disc diameter; $\mathrm{PPV}$, pars plana vitrectomy; $\mathrm{SF}_{6}$, sulfahexafluoride; $\mathrm{SMH}$, submacular hemorrhage; tPA, tissue plasminogen activator; VEGF, vascular endothelial growth factor. 


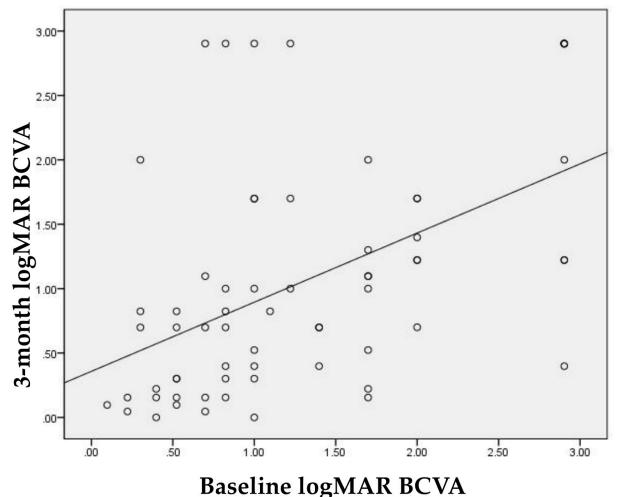

(a)

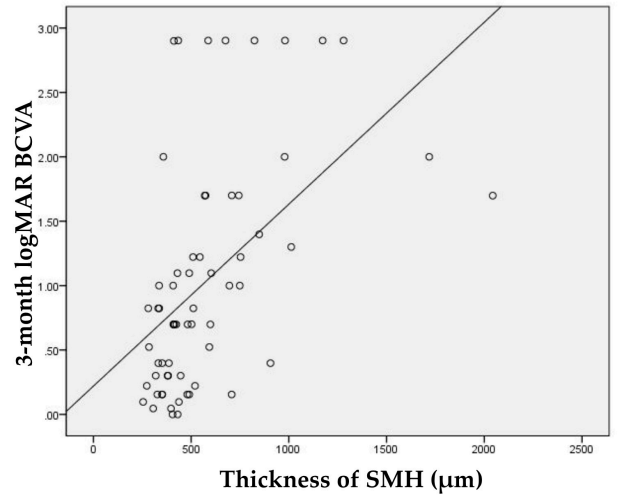

(c)

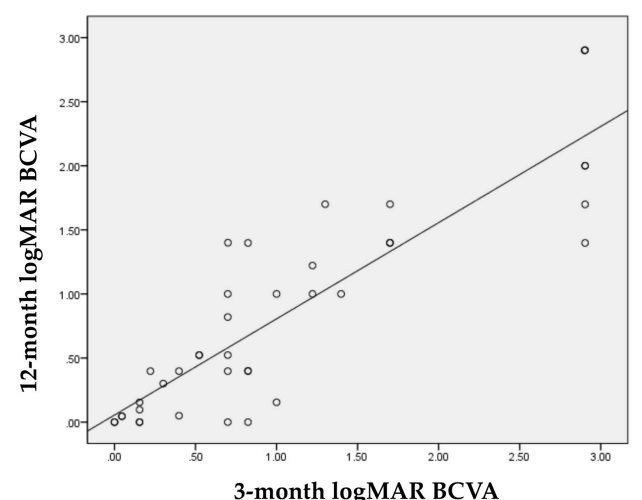

(b)

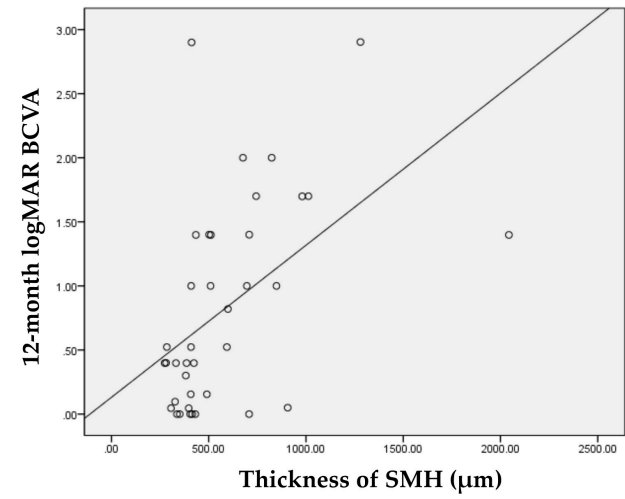

(d)

Figure 2. Scatter plots comparing (a) baseline BCVA and postoperative BCVA at 3 months and (b) BCVA at 3 months and at 12 months, as well as SMH thickness and (c) BCVA at 3 months and (d) BCVA at 12 months. BCVA, best-corrected visual acuity; LogMAR, logarithm of the minimum angle of resolution; $\mathrm{SMH}$, submacular hemorrhage.

\section{Discussion}

In this study, we compared three treatment modalities for AMD complicated by an SMH. We included an extended spectrum of hemorrhage sizes as well as a larger number of patients than previous studies. Moreover, we divided our cases according to the SMH size and analyzed the treatment outcomes of three different modalities to identify their effects in each subgroup with homogeneity in terms of size.

The overall mean BCVA improved significantly from the baseline during the 12 month follow-up period. However, different outcomes were observed according to the SMH size and treatment modalities. In the small-sized group, anti-VEGF monotherapy was performed on the majority of patients $(51.1 \%)$ and it significantly improved the visual acuity. The two other treatment modalities also increased the mean BCVA and the magnitude of improvement showed no difference among the treatment modalities. This result may be related to the anatomical outcome that a small-sized SMH was effectively regressed by anti-VEGF only (75\% of complete regression) without gas or vitrectomy. Consistent with our results, previous studies reported the efficacy of anti-VEGF monotherapy with a minimally invasive technique $[6,7,9,18,19]$. This result suggests that a small-sized SMH could be resolved with anti-VEGF only, even in the absence of additional treatments accelerating the displacement of the hemorrhage.

However, anti-VEGF monotherapy was no longer effective in the medium-sized group while the other two modalities increased the mean BCVA. Moreover, anatomical outcomes were worse with monotherapy ( $33 \%$ of complete regression) than with other modalities $(67 \%$ and $83 \%$ of complete displacement, respectively). In a retrospective study comparing anti-VEGF monotherapy with a combination of anti-VEGF, tPA and gas [19], the combination therapy groups showed better outcomes than the monotherapy group (mean changes of +1 Snellen line vs. 0 line). A more recent study comparing ranibizumab monotherapy with 
a combination of PD with ranibizumab [20] reported that a combination therapy with a higher proportion of 0.3 lines or more visual improvement tended to be more effective than monotherapy ( $57.1 \%$ vs. $37.9 \%$ ) although mean changes in BCVA showed no statistical difference between groups. Thinner and smaller hemorrhages were reported in their study than in our study $(269 \mu \mathrm{m}$ vs. $792 \mu \mathrm{m}$ in the medium-sized group and 8.2 disc area [DA] vs. 15.3-20.3 DA in the medium-sized group when the SMH area was recalculated from the ODD) and might cause no significant difference between the treatment modalities. Shin et al. [21] compared the combination of PD with anti-VEGF and anti-VEGF monotherapy. Although both groups achieved a significant improvement in BCVA at 6 months, the short-term visual outcomes at 1 month were better in the combination group than in the monotherapy group, suggesting different regression rates between the groups. Their SMH size was also smaller than that in our study (9.3 DA when it was recalculated vs. 15.3-20.3 DA). Thick and large SMHs may not be absorbed efficiently with anti-VEGF only, which limits the visual improvement and requires additional treatment options to displace the hemorrhage from the fovea.

In this study, the only effective treatment option for visual improvement in the large-sized SMH was the PPV/tPA/SF 6 . Limited studies have reported the efficacy of a vitrectomy and subretinal tPA for SMH [5]. Fassbender et al. reported that the PPV/tPA/SF 6 showed a better outcome than PD only (changes of mean BCVA were -0.90 vs. +0.10 logMAR units) [22]. Treumer et al. treated SMH patients with a PPV, a subretinal tPA and anti-VEGF [23]. The mean SMH size was 4.3 ODD (range 1.5-15) and the mean thickness was $762 \mu \mathrm{m}$ (range 217-1840 $\mu \mathrm{m}$ ), similar to those in our study. They reported complete displacement of the $\mathrm{SMH}$ in $87 \%$ of eyes. In this study, the high percentage of complete displacement in the large-sized group was achieved with a PPV/tPA/SF 6 (86\%) but not with $\mathrm{C}_{3} \mathrm{~F}_{8} /$ anti-VEGF (25\%). This result suggests that for a massive $\mathrm{SMH}$, a vitrectomy with a subretinal tPA is more efficient than $\mathrm{PD}$, showing better functional and anatomical outcomes.

The amount of the SMH that has been considered a prognostic factor for visual outcomes [23] is clinically measured in terms of thickness or diameter. The duration of the $\mathrm{SMH}$ is also a prognostic factor in a SMH [24] although several studies have reported contrasting results [11,25]. In this study, we found a positive correlation between the SMH thickness and visual outcomes. Additionally, eyes with a hemorrhage duration $\leq 14$ days showed better visual outcomes than those with a duration $>14$ days. Thus, the rapid removal of a hemorrhage within 14 days is important for patients with a massive SMH.

In this study, a strong correlation of BCVA at 3 months with the BCVA at 12 months was identified, suggesting that optimal initial treatment and subsequent anti-VEGF retreatment may be helpful to maintain the initial visual gain. Treumer et al. [23] reported a stronger positive correlation between the 3 month and final BCVA than between the baseline and final BCVA, similar to that in our study. They adopted strict retreatment regimens with anti-VEGF based on OCT and functional criteria [26]. After the initial treatment, we also administered additional anti-VEGFs as needed based on OCT findings, which may have contributed to the maintenance of visual acuity.

Of the complications during the follow-up, vitreous hemorrhage was more common in the large-sized group (36.4\%) than in other groups (6.7\% and $4.8 \%$, respectively). However, the percentage of vitreous hemorrhage was not different among the treatment modalities (10\%, $12 \%$ and $9 \%$, respectively). Although the PPV/tPA/SF 6 was a more invasive technique, intraoperative and other postoperative complications were rarely observed. One case of an RPE rip (4.3\%), one of a macular hole (4.3\%) and none of retinal detachment (RD) were observed, similar to that in previous studies (3.4 12\% of RPE rip, $2.4 \sim 4.2 \%$ of macular hole and $2.4 \sim 8.3 \%$ of $\mathrm{RD}$ ) [27-29]. This may be partially attributable to the small number of patients treated with a PPV/tPA/SF 6 . All procedures were done by an experienced surgeon, which minimized complications. It was also helpful to try to find a suitable location for the subretinal injection location while avoiding large blood vessels and pigment epithelial detachment using multimodal images including fundus photography and OCT. The rarity of complications associated with the PPV/tPA/SF 6 and its efficacy in the large-sized hemorrhage group could justify our treatment selection. 
Our study has several limitations. Due to the retrospective characteristics, the treatment modalities were not consistently applied in each group according to the SMH size, resulting selection bias. However, all judgments were made by one experienced surgeon to minimize bias in the choice of treatment options. In addition, due to the nature of the disease, the number of patients with a large SMT was small, which limited the statistical analysis. The scarcity of the disease and the fact that inadequate treatment in time can lead to severe vision loss make it difficult to conduct large-scale prospective studies for a limited period along with ethical issues. Therefore, in the study of rare diseases, the real-world data including many patients can be more important.

In conclusion, the individualized treatment options for a SMH depending on the hemorrhage size showed acceptable anatomical outcomes with visual improvement in each subgroup. For the small-sized SMH, anti-VEGF monotherapy was useful. However, in the medium-sized SMH, anti-VEGF only was not effective and the rapid removal of the hemorrhage was required for a thick SMH. For a large-sized SMH, a vitrectomy with a subretinal tPA and expansile gas were the only effective treatment options. Our strategy of selecting the treatment modality according to the hemorrhage size could be a reference for the treatment of SMHs.

Author Contributions: Design and conduct of the study (M.S.); collection and management of data (S.J., D.-G.P., M.S.); analysis and interpretation of data (S.J., D.-G.P., M.S.); preparation, review and approval of the manuscript (S.J., D.-G.P., M.S.). All authors have read and agreed to the published version of the manuscript.

Funding: This work was supported by the 2018 Yeungnam University research grant (218A480006).

Conflicts of Interest: The authors declare no conflict of interest.

\section{References}

1. Glatt, H.; Machemer, R. Experimental subretinal hemorrhage in rabbits. Am. J. Ophthalmol. 1982, 94, 762-773. [PubMed]

2. Avery, R.L.; Fekrat, S.; Hawkins, B.S.; Bressler, N.M. Natural history of subfoveal subretinal hemorrhage in age-related macular degeneration. Retina 1996, 16, 183-189. [PubMed]

3. Rijken, D.C. Plasminogen activators and plasminogen activator inhibitors: Biochemical aspects. Bailliere Clin. Haematol. 1995, 8, 291-312.

4. Soliman, W.; Lund-Andersen, H.; Larsen, M. Resolution of subretinal haemorrhage and fluid after intravitreal bevacizumab in aggressive peripapillary subretinal neovascularization. Acta Ophthalmol. Scand. 2006, 84, 707-708.

5. Stanescu-Segall, D.; Balta, F.; Jackson, T.L. Submacular hemorrhage in neovascular age-related macular degeneration: A synthesis of the literature. Surv. Ophthalmol. 2016, 61, 18-32.

6. Chang, M.A.; Do, D.V.; Bressler, S.B.; Cassard, S.D.; Gower, E.W.; Bressler, N.M. Prospective one-year study of ranibizumab for predominantly hemorrhagic choroidal neovascular lesions in age-related macular degeneration. Retina 2010, 30, 1171-1176.

7. Cho, H.J.; Koh, K.M.; Kim, H.S.; Lee, T.G.; Kim, C.G.; Kim, J.W. Anti-vascular endothelial growth factor monotherapy in the treatment of submacular hemorrhage secondary to polypoidal choroidal vasculopathy. Am. J. Ophthalmol. 2013, 156, 524-531.

8. Spaide, R.F.; Fisher, Y.L. Intravitreal bevacizumab (Avastin) treatment of proliferative diabetic retinopathy complicated by vitreous hemorrhage. Retina 2006, 26, 275-278.

9. Shienbaum, G.; Garcia Filho, C.A.; Flynn, H.W., Jr.; Nunes, R.P.; Smiddy, W.E.; Rosenfeld, P.J. Management of submacular hemorrhage secondary to neovascular age-related macular degeneration with anti-vascular endothelial growth factor monotherapy. Am. J. Ophthalmol. 2013, 155, 1009-1013.

10. Irvine, W.D.; Johnson, M.W.; Hernandez, E.; Olsen, K.R. Retinal toxicity of human tissue plasminogen activator in vitrectomized rabbit eyes. Arch. Ophthalmol. 1991, 109, 718-722.

11. Hassan, A.S.; Johnson, M.W.; Schneiderman, T.E.; Regillo, C.D.; Tornambe, P.E.; Poliner, L.S.; Blodi, B.A.; Elner, S.G. Management of submacular hemorrhage with intravitreous tissue plasminogen activator injection and pneumatic displacement. Ophthalmology 1999, 106, 1900-1906. [PubMed]

12. Johnson, M.W.; Olsen, K.R.; Hernandez, E. Tissue plasminogen activator treatment of experimental subretinal hemorrhage. Retina 1991, 11, 250-258. [PubMed] 
13. Ohji, M.; Saito, Y.; Hayashi, A.; Lewis, J.M.; Tano, Y. Pneumatic displacement of subretinal hemorrhage without tissue plasminogen activator. Arch. Ophthalmol. 1998, 116, 1326-1332. [PubMed]

14. Stifter, E.; Michels, S.; Prager, F.; Georgopoulos, M.; Polak, K.; Hirn, C.; Schmidt-Erfurth, U. Intravitreal bevacizumab therapy for neovascular age-related macular degeneration with large submacular hemorrhage. Am. J. Ophthalmol. 2007, 144, 886-892. [PubMed]

15. Al-Hity, A.; Steel, D.H.; Yorston, D.; Gilmour, D.; Koshy, Z.; Young, D.; Hillenkamp, J.; McGowan, G. Incidence of submacular haemorrhage (SMH) in Scotland: A Scottish Ophthalmic Surveillance Unit (SOSU) study. Eye 2019, 33, 486-491. [PubMed]

16. Chang, Y.S.; Kim, J.H.; Kim, J.W.; Kim, C.G.; Lee, D.W. Development of submacular hemorrhage in neovascular age-related macular degeneration: Influence on visual prognosis in a clinical setting. Korean J. Ophthalmol. 2018, 32, 361-368.

17. Klettner, A.; Groteluschen, S.; Treumer, F.; Roider, J.; Hillenkamp, J. Compatibility of recombinant tissue plasminogen activator (rtPA) and aflibercept or ranibizumab coapplied for neovascular age-related macular degeneration with submacular haemorrhage. Br. J. Ophthalmol. 2015, 99, 864-869.

18. Kim, J.H.; Chang, Y.S.; Kim, J.W.; Kim, C.G.; Yoo, S.J.; Cho, H.J. Intravitreal anti-vascular endothelial growth factor for submacular hemorrhage from choroidal neovascularization. Ophthalmology 2014, 121, 926-935.

19. Sacu, S.; Stifter, E.; Vecsei-Marlovits, P.V.; Michels, S.; Schutze, C.; Prunte, C.; Schmidt-Erfurth, U. Management of extensive subfoveal haemorrhage secondary to neovascular age-related macular degeneration. Eye 2009, 23, 1404-1410.

20. Cho, H.J.; Koh, K.M.; Kim, J.H.; Kim, H.S.; Han, J.I.; Lew, Y.J.; Lee, T.G.; Kim, J.W. Intravitreal ranibizumab injections with and without pneumatic displacement for treating submacular hemorrhage secondary to neovascular age-related macular degeneration. Retina 2015, 35, 205-212.

21. Shin, J.Y.; Lee, J.M.; Byeon, S.H. Anti-vascular endothelial growth factor with or without pneumatic displacement for submacular hemorrhage. Am. J. Ophthalmol. 2015, 159, 904-914. [CrossRef] [PubMed]

22. Fassbender, J.M.; Sherman, M.P.; Barr, C.C.; Schaal, S. Tissue plasminogen activator for subfoveal hemorrhage due to age-related macular degeneration: Comparison of 3 treatment modalities. Retina 2016, 36, 1860-1865. [CrossRef] [PubMed]

23. Treumer, F.; Wienand, S.; Purtskhvanidze, K.; Roider, J.; Hillenkamp, J. The role of pigment epithelial detachment in AMD with submacular hemorrhage treated with vitrectomy and subretinal co-application of rtPA and anti-VEGF. Graefe Arch. Clin. Exp. Ophthalmol. 2017, 255, 1115-1123. [CrossRef] [PubMed]

24. Hattenbach, L.O.; Klais, C.; Koch, F.H.; Gumbel, H.O. Intravitreous injection of tissue plasminogen activator and gas in the treatment of submacular hemorrhage under various conditions. Ophthalmology 2001, 108, 1485-1492. [CrossRef]

25. Schulze, S.D.; Hesse, L. Tissue plasminogen activator plus gas injection in patients with subretinal hemorrhage caused by age-related macular degeneration: Predictive variables for visual outcome. Graefe Arch. Clin. Exp. Ophthalmol. 2002, 240, 717-720. [CrossRef] [PubMed]

26. Gerding, H.; Loukopoulos, V.; Riese, J.; Hefner, L.; Timmermann, M. Results of flexible ranibizumab treatment in age-related macular degeneration and search for parameters with impact on outcome. Graefe Arch. Clin. Exp. Ophthalmol. 2011, 249, 653-662. [CrossRef]

27. Treumer, F.; Roider, J.; Hillenkamp, J. Long-term outcome of subretinal coapplication of rtPA and bevacizumab followed by repeated intravitreal anti-VEGF injections for neovascular AMD with submacular haemorrhage. Br. J. Ophthalmol. 2012, 96, 708-713. [CrossRef]

28. Sharma, S.; Kumar, J.B.; Kim, J.E.; Thordsen, J.; Dayani, P.; Ober, M.; Mahmoud, T.H. Pneumatic displacement of submacular hemorrhage with subretinal air and tissue plasminogen activator: Initial United States experience. Ophthalmol. Retina 2018, 2, 180-186.

29. Hillenkamp, J.; Surguch, V.; Framme, C.; Gabel, V.P.; Sachs, H.G. Management of submacular hemorrhage with intravitreal versus subretinal injection of recombinant tissue plasminogen activator. Graefe Arch. Clin. Exp. Ophthalmol. 2010, 248, 5-11. [CrossRef]

(C) 2020 by the authors. Licensee MDPI, Basel, Switzerland. This article is an open access article distributed under the terms and conditions of the Creative Commons Attribution (CC BY) license (http://creativecommons.org/licenses/by/4.0/). 